\title{
Prevalence of Staphylococcus aureus and Use of Antistaphylococcal Therapy in Children Hospitalized with Pneumonia
}

\author{
Jennifer M. Frush, BA', Yuwei Zhu, MD, MS², Kathryn M. Edwards, MD³, Carlos G. Grijalva, MD, MPH4, \\ Isaac P. Thomsen, MD, MSCl${ }^{3}$, Wesley H. Self, MD, MPH'5, Seema Jain, $\mathrm{MD}^{6}$, Evan J. Anderson, MD ${ }^{7}$, Krow Ampofo, MD', \\ Andrew T. Pavia, MD', Sandra R. Arnold MD', Jonathan A. McCullers MD, Derek J. Williams, MD, MPH ${ }^{10 *}$
}

\begin{abstract}
${ }^{1}$ Duke University School of Medicine, Durham, North Carolina; ${ }^{2}$ Department of Biostatistics, Vanderbilt University School of Medicine, Nashville, Tennessee; ${ }^{3}$ Division of Infectious Diseases, Monroe Carell Jr. Children's Hospital and the Department of Pediatrics, Vanderbilt University School of Medicine, Nashville, Tennessee; ${ }^{4}$ Department of Health Policy, Vanderbilt University School of Medicine, Nashville, Tennessee; ${ }^{5}$ Department of Emergency Medicine, Vanderbilt University School of Medicine, Nashville, Tennessee; ${ }^{6}$ Centers for Disease Control and Prevention, Atlanta, Georgia; 'Division of Infectious Diseases, Departments of Medicine and Pediatrics, Emory University School of Medicine, Atlanta, Georgia; ${ }^{8}$ Division of Infectious Diseases, Primary Children's Medical Center and the Department of Pediatrics, University of Utah School of Medicine, Salt Lake City, Utah; ${ }^{9}$ Division of Infectious Diseases, LeBonheur Children's Hospital and the Department of Pediatrics, University of Tennessee Health Sciences Center, Memphis, Tennessee; ${ }^{10}$ Division of Hospital Medicine, Monroe Carell Jr. Children's Hospital and the Department of Pediatrics, Vanderbilt University School of Medicine, Nashville, Tennessee.
\end{abstract}

Within a cohort of $>2,000$ children hospitalized with community-acquired pneumonia, staphylococcal pneumonia was rare (1\%) but associated with adverse in-hospital outcomes. Despite this low prevalence, use of antistaphylococcal antibiotics was common (24\%). Efforts are needed to minimize overuse of antistaphylococcal antibiotics while also ensuring adequate treatment for pathogen-specific diseases. Journal of Hospital Medicine 2018;13:848-852. Published online first October 31, 2018. ( 2018 Society of Hospital Medicine

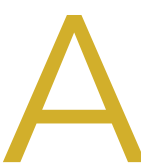

Ithough Staphylococcus aureus pneumonia is common in children with cystic fibrosis and those with healthcare-associated infections (eg, ventilator-associated pneumonia), ${ }^{1,2} \mathrm{~S}$. aureus is an uncommon cause of community-acquired pneumonia in children. In recent years, concerns have arisen about the increasing frequency and severity of staphylococcal pneumonia, largely fueled by the emergence of community-associated methicillin-resistant S. aureus (MRSA) ${ }^{3,4}$ Thus, therapy with clindamycin or vancomycin, both active against MRSA, has been recommended when $S$. aureus is suspected. ${ }^{5}$ Given the lack of rapid and sensitive approaches to the detection of the etiologies of pneumonia, antibiotic selection is most often empirical, contributing to overuse of anti-MRSA antibiotics. In addition, resistance against these antibiotics, especially clindamycin, has been increasing. ${ }^{6,7}$

A better understanding of the likelihood of staphylococcal pneumonia would help to optimize empirical antibiotic selection, allowing for judicious use of antistaphylococcal antibiotics, while also avoiding poor outcomes due to delays in effective treatment when $S$. aureus is present. ${ }^{8}$ Using data from

*Address for correspondence: Derek J. Williams, MD, MPH, Vanderbilt University Medical Center, DOT11205, 2200 Children's Way, Nashville, TN 372329000; E-mail: derek.williams@vumc.org

Received: June 4, 2018; Revised: August 21, 2018;

Accepted: September 3, 2018

@2018 Society of Hospital Medicine DOI 10.12788/jhm.3093 a multicenter, population-based study of pneumonia hospitalizations in children, we sought to describe the prevalence, clinical characteristics, and in-hospital outcomes of staphylococcal pneumonia and the prevalence of antistaphylococcal antibiotic use.

\section{METHODS}

The Etiology of Pneumonia in the Community (EPIC) study was a prospective, active, population-based surveillance study of pneumonia hospitalizations among children (age $<18$ years) conducted between 2010 and 2012 at three children's hospitals, including two in Tennessee and one in Utah. ${ }^{9}$ Children hospitalized with clinical evidence of pneumonia and radiographic evidence confirmed by a blinded review by study radiologists were enrolled. Etiologic assessments included blood analysis for bacterial culture, serology for eight respiratory viruses, pneumococcal and group A streptococcal polymerase chain reaction (PCR), and naso/oro-pharyngeal swabs for PCR for 13 respiratory viruses, Mycoplasma pneumoniae, and Chlamydophila pneumoniae. Data from other clinical specimens (pleural fluid, high-quality endotracheal aspirate, or quantified bronchoalveolar lavage fluid) were also recorded. For this study, we included only children with at least one bacterial culture and complete information about antibiotic use. Those with confirmed fungal pneumonia were excluded. Additional details regarding the study population and methods have been published previously.

Staphylococcal pneumonia was defined based on the detection of S. aureus by culture (any site) or PCR (pleural fluid 
only), regardless of codetection of other pathogens. Antibiotic susceptibility profiles were used to classify $S$. aureus isolates as MRSA or methicillin-sensitive $S$. aureus (MSSA). The remaining children were classified as nonstaphylococcal pneumonia including children with other bacterial pathogens detected (classified as other bacterial pneumonia, excludes atypical bacteria), atypical bacteria, viruses, and no pathogens detected.

Use of anti-MRSA antibiotics (vancomycin, clindamycin, linezolid, doxycycline, and trimethoprim-sulfamethoxazole) and any antistaphylococcal antibiotics (anti-MRSA agents plus oxacillin, nafcillin, and cefazolin) during and after the first two calendar days of admission was identified by medical record review.

Descriptive statistics included number (\%) and median (interquartile range, [IQR]) for categorical and continuous variables, respectively. Baseline clinical characteristics and outcomes were compared between children with staphylococcal versus nonstaphylococcal pneumonia, those with staphylococcal versus other bacterial pneumonia, and those with MRSA versus MSSA pneumonia using Wilcoxon rank-sum and Pearson's chisquare tests where appropriate. To account for multiple comparisons, we used a Bonferroni corrected $P$ value threshold of $<.001$ to determine statistical significance.

\section{RESULTS}

Of the 2,358 children enrolled in the EPIC study hospitalized with radiographically confirmed pneumonia, 2,146 (91.0\%) had $\geq 1$ bacterial culture obtained. Two children with Histoplasma capsulatum fungal infection and six children with incomplete antibiotic utilization data were excluded, yielding a final study population of 2,138 children. Among these, blood samples were obtained from 2,134 (>99\%) children for culture, pleural fluid from 87 (4\%) children, bronchoalveolar lavage fluid from 31 (1\%) children, and endotracheal aspirate from 80 (4\%) children. Across all culture types, there were 2,332 initial cultures; 2,150 (92\%) were collected within the first 24 hours.

Staphylococcal pneumonia was detected in 23 of the 2,138 children (1\% [95\% Cl 0.7, 1.6]; 17 MRSA, 6 MSSA). Of these, $6 / 23$ (26\%) had bacteremia, 12/23 (52\%) had a positive pleural fluid, and 9/23 (39\%) had a positive culture from bronchoalveolar lavage fluid or endotracheal aspirate; 4/23 (17\%) children had $S$. aureus detected from more than one site. Three children (13\%) with S. aureus had a viral codetection, including two with influenza.

Compared with children with nonstaphylococcal pneumonia, those with staphylococcal pneumonia were more likely to have a parapneumonic effusion (78\% vs 12\%, $P<.001)$, but less likely to have cough (78\% vs 95\%, $P<.001)$. Other baseline characteristics were similar between the two groups. Children with staphylococcal pneumonia had more adverse outcomes than those without (Table), including longer median length of stay (10 vs 3 days, $P<.001$ ), more frequent admission to intensive care $(83 \%$ vs $21 \%, P<.001)$, and more frequent invasive mechanical ventilation (65\% vs 7\%, $P<.001$ ). Similar findings were noted when staphylococcal pneumonia was compared with pneumonia caused due to other bacterial pathogens ( $\mathrm{n}$
= 124). There were no significant differences in baseline characteristics or clinical course between children with MRSA and MSSA pneumonia, although the numbers were small. Overall, S. aureus was detected in $18 / 267(7 \%)$ children with parapneumonic effusion and 19/462 (4\%) children admitted to intensive care. Importantly, there were no confirmed S. aureus cases among children with less severe pneumonia, defined as lacking both parapneumonic effusion and intensive care admission ( $n=1,488)$.

Overall, 519 children (24\%) received antistaphylococcal therapy during their hospitalization (512/519, 99\% received anti-MRSA therapy), including 22 of the 23 children with $S$. aureus detected (the only child without antistaphylococcal therapy had $S$. aureus detected from a high-quality endotracheal tube aspirate only and also had respiratory syncytial virus detected). Clindamycin was most often used ( $n=266,51 \%$ ), followed by vancomycin ( $n=128,24 \%)$, clindamycin plus vancomycin ( $n=$ $83,16 \%)$, and others ( $n=42,8 \%)$. During the first two days of hospitalization, 479 children (22\%) received antistaphylococcal therapy (477 received anti-MRSA therapy). After the first two days, 351 children (16\%) received antistaphylococcal therapy (346/351, 99\% received anti-MRSA therapy). Use of antistaphylococcal therapy was very common in those admitted to intensive care (182/462, 39\%; all but two received anti-MRSA therapy) and in those requiring invasive mechanical ventilation (103/159, 65\%). Among those lacking both parapneumonic effusion and intensive care admission ( $n=1,488), 232(16 \%)$ received antistaphylococcal therapy.

\section{DISCUSSION}

In our large, population-based study of $>2,000$ children hospitalized with community-acquired pneumonia, S. aureus was identified in only $1 \%$ of children. Compared with children with other pneumonia etiologies, staphylococcal pneumonia was associated with increased disease severity. Among the small numbers studied, no differences in outcomes were found between children with MRSA and MSSA disease. Despite the low prevalence of staphylococcal pneumonia, almost one in four children received antistaphylococcal antibiotic therapy; anti-MRSA therapy was used almost exclusively.

The severity of staphylococcal pneumonia was striking, with $>80 \%$ of children with $S$. aureus detected being admitted to intensive care, about $65 \%$ requiring invasive mechanical ventilation, and $>75 \%$ with parapneumonic effusion. These findings are similar to those of prior retrospective studies. ${ }^{4,10}$ The association between staphylococcal pneumonia and adverse outcomes underscores the importance of prompt institution of antimicrobial therapy targeting $S$. aureus in high-risk patients. This is noteworthy given recent epidemiological data demonstrating increases in MSSA relative to MRSA infections in children, ${ }^{6}$ and the known superiority of beta-lactam versus vancomycin for MSSA infections, including pneumonia. ${ }^{11}$

Although detection of staphylococcal infection was rare, almost a quarter of children received antistaphylococcal therapy; nearly all of these children received anti-MRSA therapy. Confirming a bacterial etiology of pneumonia, however, is 
TABLE. Clinical Characteristics, Outcomes, and Antibiotic Use among Children Hospitalized with Staphylococcal and Nonstaphylococcal Community-Acquired Pneumonia

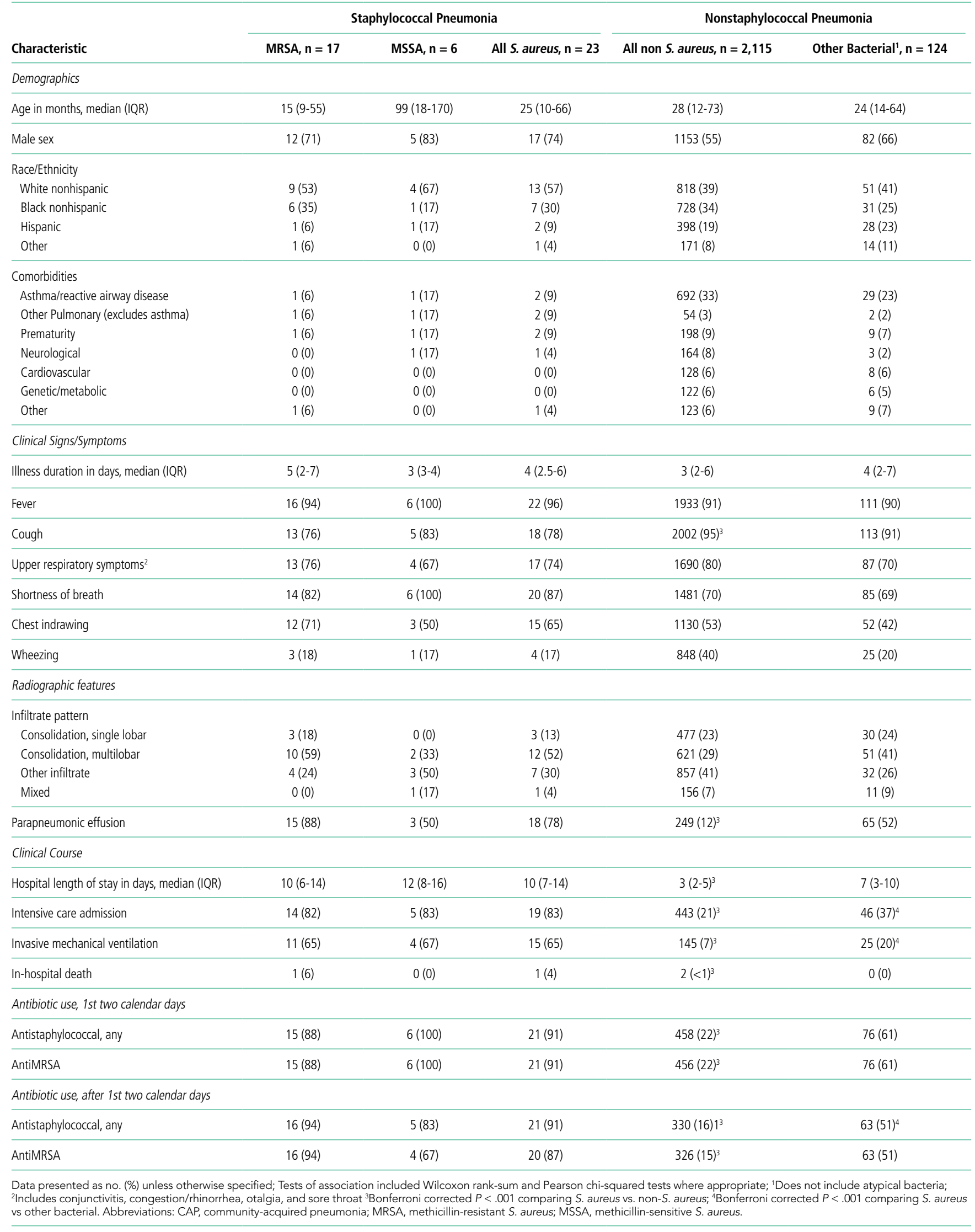


challenging. Given the severity associated with staphylococcal pneumonia, it is not surprising that use of antistaphylococcal therapy outpaced staphylococcal detections. Antistaphylococcal therapy was especially common in those with severe pneumonia, suggesting that disease severity is an important factor that influences initial antibiotic treatment decisions. Even so, two children with MRSA detected did not initially receive anti-MRSA therapy, highlighting the challenge of balancing judicious antibiotic selection along with ensuring effective treatment. Perhaps more striking is the finding that $16 \%$ of children received antistaphylococcal therapy beyond the first two days of hospitalization, presumably after the initial culture results were available. This suggests that clinicians are reluctant to stop antistaphylococcal therapy when the etiology is unknown, although certain features, such as negative cultures, rapid clinical improvement, and lack of risk factors for staphylococcal disease, may provide important clues to support de-escalation of empiric antibiotic therapy. It is also possible that some antibiotics with antistaphylococcal activity were used for alternative indications (eg, clindamycin for penicillin allergy or concern for aspiration pneumonia).

A simple strategy for tailoring antibiotic treatment is maximizing opportunities to identify a causative pathogen. Despite the very low yield of blood cultures in children with pneumonia overall, bacteremia is more common in children with severe pneumonia and those with parapneumonic effusion, especially when cultures are obtained prior to antibiotic use. ${ }^{12,13}$ Similarly, obtaining pleural fluid is often therapeutic and significantly improves the chances of identifying a bacterial pathogen. ${ }^{14}$ Moreover, at least one study suggests that $S$. aureus is much less likely in cases of culture-negative parapneumonic effusions. ${ }^{15}$ Institutional guidelines, order sets, and antimicrobial stewardship teams are also effective strategies that can facilitate judicious antibiotic use. In particular, stewardship experts can be very useful in assisting clinicians around de-escalation of therapy. ${ }^{16}$ Use of procalcitonin, a biomarker associated with bacterial infections, ${ }^{17}$ and prognostic tools to identify risk for adverse outcomes, ${ }^{18}$ may also inform treatment decisions and are deserving of further study.

Our study must be considered in the light of its strengths and limitations. Analysis was derived from a population-based surveillance study of community-acquired pneumonia hospitalizations in three children's hospitals and may not be generalizable to other settings. Nevertheless, the antibiotic-prescribing practices identified in our study are consistent with those from a larger network of children's hospitals in the United States. ${ }^{19}$ The relatively small number of children with $S$. aureus identified limited our ability to control for potential confounding factors. Some cases of staphylococcal pneumonia may not have been identified. All study children, however, were prospectively enrolled and had samples systematically collected and tested for etiology, likely leading to few cases of misclassification for this pathogen.

Our study demonstrates a very low prevalence of $S$. aureus detection among children hospitalized with pneumonia and highlights the association between staphylococcal disease and adverse in-hospital outcomes. We also document important discrepancies between disease prevalence and utilization of antistaphylococcal therapy, especially anti-MRSA therapy. Improved approaches are needed to minimize overuse of antistaphylococcal antibiotics while also ensuring adequate therapy for those who need it.

Disclosures: Drs. Zhu, Edwards, Self, Ampofo, Arnold, McCullers, and Williams report grants from the Centers for Disease Control and Prevention during the conduct of the study. Ms. Frush has nothing to disclose. Dr. Jain has nothing to disclose. Dr. Grijalva reports other from Merck, grants and other from Sanofi, other from Pfizer, grants from $\mathrm{CDC}$, grants from $\mathrm{AHRQ}$, grants from $\mathrm{NIH}$, and grants from Campbell Alliance, outside the submitted work. Dr. Self reports grants from $\mathrm{CDC}$, during the conduct of the study; personal fees from Cempra Pharmaceuticals, grants and personal fees from Ferring Pharmaceuticals, personal fees from BioTest AG, personal fees from Abbott Point of Care, personal fees from Gilead Pharmaceuticals, personal fees from Pfizer, grants from Merck, outside the submitted work. Dr. Thomsen has nothing to disclose. Dr. Ampofo reports grants from $C D C$, during the conduct of the study; other from GlaxoSmithKline, other from Cubist Pharmaceuticals outside the submitted work; and KA collaborate with BioFire Diagnostics, Inc. (formerly Idaho Technology, Inc.) on several NIH grants. Dr. Pavia reports grants from NAID/NIH, grants from NAID/NIH, grants from CDC, personal fees from WebMD, personal fees from Antimicrobial Therapy Inc., outside the submitted work.

Funding: This work was supported by the National Institute of Allergy and Infectious Diseases of the National Institutes of Health under Award Number K23Al104779 to D.J.W. and Award 1K23Al113150 to I.P.T., the National Institute of General Medical Sciences under Award K23GM110469 to W.H.S., and the Agency for Healthcare Research and Quality under Award R03HSO22342 to C.G.G. The EPIC study was supported by the Influenza Division in the National Center for Immunizations and Respiratory Diseases at the Centers for Disease Control and Prevention through cooperative agreements with each study site and was based on a competitive research funding opportunity. The findings and conclusions in this report are those of the authors and do not necessarily represent the views of the National Institute of Allergy and Infectious Diseases, the National Institute of General Medical Sciences, the Agency for Healthcare Research and Quality, or the Centers for Disease Control and Prevention.

\section{References}

1. Akil N, Muhlebach MS. Biology and management of methicillin resistant Staphylococcus aureus in cystic fibrosis. Pediatr Pulmonol. 2018. doi: 10.1002/ppul.24139

2. Srinivasan R, Asselin J, Gildengorin G, Wiener-Kronish J, Flori HR. A prospective study of ventilator-associated pneumonia in children. Pediatrics. 2009;123(4):1108-1115. doi: 10.1542/peds.2008-1211.

3. Gonzalez BE, Martinez-Aguilar G, Hulten KG, et al. Severe Staphylococcal sepsis in adolescents in the era of community-acquired methicillin-resistant Staphylococcus aureus. Pediatrics. 2005;115(3):642-648. doi: 10.1542/ peds.2004-2300

4. Carrillo-Marquez MA, Hulten KG, Hammerman W, Lamberth L, Mason EO, Kaplan SL. Staphylococcus aureus pneumonia in children in the era of community-acquired methicillin-resistance at Texas Children's Hospital. Pediatr Infect Dis J. 2011;30(7):545-550. doi: 10.1097/INF.0b013e31821618be.

5. Bradley JS, Byington CL, Shah SS, et al. The management of community-acquired pneumonia in infants and children older than 3 months of age: clinical practice guidelines by the pediatric infectious diseases society and the infectious diseases society of America. Clin Infect Dis. 2011;53(7):e25-e76. doi: 10.1093/cid/cir531.

6. Sutter DE, Milburn E, Chukwuma U, Dzialowy N, Maranich AM, Hospenthal DR. Changing susceptibility of Staphylococcus aureus in a US pediatric population. Pediatrics. 2016;137(4):e20153099-e20153099. doi: 10.1542/ peds.2015-3099

7. Sakoulas G, Moellering RC, Jr. Increasing antibiotic resistance among methicillin-resistant Staphylococcus aureus strains. Clin Infect Dis. 2008;46(Suppl 5):S360-S367. doi: 10.1086/533592.

8. Rubinstein E, Kollef MH, Nathwani D. Pneumonia caused by methicillin-resistant Staphylococcus aureus. Clin Infect Dis. 2008;46(Suppl 5):S378-S385. 
doi: $10.1086 / 533594$

9. Jain S, Williams DJ, Arnold SR, et al. Community-acquired pneumonia requiring hospitalization among U.S. children. N Engl J Med. 2015;372(9):835845. doi: 10.1056/NEJMoa1405870.

10. Kallen AJ, Reed C, Patton M, Arnold KE, Finelli L, Hageman J. Staphylococcus aureus community-onset pneumonia in patients admitted to children's hospitals during autumn and winter of 2006-2007. Epidemiol Infect. 2010;138(5):666-672. doi: 10.1017/S095026880999135X

11. González C, Rubio M, Romero-Vivas J, González M, Picazo JJ. Bacteremic pneumonia due to Staphylococcus aureus: A comparison of disease caused by methicillin-resistant and methicillin-susceptible organisms. Clin Infect Dis. 1999;29(5):1171-1177. doi: 10.1086/313440.

12. Myers AL, Hall M, Williams DJ, et al. Prevalence of bacteremia in hospitalized pediatric patients with community-acquired pneumonia. Pediatr Infect Dis J. 2013;32(7):736-740. doi: 10.1097/INF.0b013e318290bf63.

13. Iroh Tam PY, Bernstein E, Ma X, Ferrieri P. Blood culture in evaluation of pediatric community-acquired pneumonia: A systematic review and meta-analysis. Hosp Pediatr. 2015;5(6):324-336. doi: 10.1542/hpeds.2014-0138.

14. Byington $C L$, Spencer $L Y$, Johnson $T A$, et al. An epidemiological investigation of a sustained high rate of pediatric parapneumonic empyema: risk factors and microbiological associations. Clin Infect Dis. 2002;34(4):434440. doi: $10.1086 / 338460$

15. Blaschke AJ, Heyrend C, Byington CL, et al. Molecular analysis improves pathogen identification and epidemiologic study of pediatric parapneumonic empyema. Pediatr Infect Dis J. 2011;30(4):289-294. doi: 10.1097/ INF.0b013e3182002d14.

16. Banerjee R, Teng CB, Cunningham SA, et al. Randomized trial of rapid multiplex polymerase chain reaction-based blood culture identification and susceptibility testing. Clin Infect Dis. 2015;61(7):1071-1080. doi: 10.1093/ $\mathrm{cid} /$ civ447.

17. Stockmann C, Ampofo K, Killpack J, et al. Procalcitonin accurately identifies hospitalized children with low risk of bacterial community-acquired pneumonia. J Pediatr Infect Dis Soc. 2018;7(1):46-53. doi: 10.1093/jpids/ piw091.

18. Williams DJ, Zhu Y, Grijalva CG, et al. Predicting severe pneumonia outcomes in children. Pediatrics. 2016;138(4). doi: 10.1542/peds.2016-1019.

19. Brogan TV, Hall M, Williams DJ, et al. Variability in processes of care and outcomes among children hospitalized with community-acquired pneumonia. Pediatr Infect Dis J. 2012;31(10):1036-1041. doi: 10.1097/INF.0b013e$31825 f 2 b 10$ 